\title{
Genetic sequence characterization and naturally acquired immune response to Plasmodium vivax Rhoptry Neck Protein 2 (PvRON2)
}

\author{
Najara C. Bittencourt ${ }^{1}$, Juliana A. Leite', Ana Beatriz I. E. Silva², Tamirys S. Pimenta ${ }^{3}$, João Luiz Silva-Filho ${ }^{1}$, \\ Gustavo C. Cassiano', Stefanie C. P. Lopes ${ }^{4,5}$, Joao C. K. dos-Santos ${ }^{1}$, Catarina Bourgard ', Helder I. Nakaya ${ }^{6}$, \\ Ana Maria Revorêdo da Silva Ventura ${ }^{3}$, Marcus V. G. Lacerda ${ }^{4,5}$, Marcelo U. Ferreira ${ }^{7}$, Ricardo L. D. Machado ${ }^{3}$, \\ Letusa Albrecht ${ }^{2^{*}}$ and Fabio T. M. Costa ${ }^{1 *}$ (1)
}

\begin{abstract}
Background: The genetic diversity of malaria antigens often results in allele variant-specific immunity, imposing a great challenge to vaccine development. Rhoptry Neck Protein 2 (PVRON2) is a blood-stage antigen that plays a key role during the erythrocyte invasion of Plasmodium vivax. This study investigates the genetic diversity of PvRON2 and the naturally acquired immune response to $P$. vivax isolates.

Results: Here, the genetic diversity of PVRON2 $1828-2080$ and the naturally acquired humoral immune response against PVRON2 ${ }_{1828-2080}$ in infected and non-infected individuals from a vivax malaria endemic area in Brazil was reported. The diversity analysis of PVRON2 ${ }_{1828-2080}$ revealed that the protein is conserved in isolates in Brazil and worldwide. A total of 18 (19\%) patients had IgG antibodies to PvRON2 $2_{1828-2080}$. Additionally, the analysis of the antibody response in individuals who were not acutely infected with malaria, but had been infected with malaria in the past indicated that 32 patients (33\%) exhibited an IgG immune response against PVRON2.

Conclusions: PVRON2 was conserved among the studied isolates. The presence of naturally acquired antibodies to this protein in the absence of the disease suggests that PVRON2 induces a long-term antibody response. These results indicate that PVRON2 is a potential malaria vaccine candidate.
\end{abstract}

Keywords: Genetic diversity, RON2, Plasmodium vivax, Immunogenicity, Malaria

\section{Background}

Clinical manifestations of human malaria occur during the blood stage of infection by the Plasmodium parasite. Merozoites invade red blood cells in a process that involves specific interactions between parasite ligands

\footnotetext{
*Correspondence: letusa.albrecht@fiocruz.br; letusaa@gmail.com; costaftm@unicamp.br; fabiotmc72@gmail.com.br

${ }^{1}$ Laboratory of Tropical Diseases-Prof. Dr. Luiz Jacintho da Silva, Department of Genetics, Evolution, Microbiology and Immunology, University of Campinas-UNICAMP, Campinas, SP, Brazil

${ }^{2}$ Instituto Carlos Chagas, Fundação Oswaldo Cruz - FIOCRUZ, Curitiba, PR, Brazil

Full list of author information is available at the end of the article
}

and host cell receptors; the merozoites are propelled by actin/myosin motors through the moving junction (MJ). The $M J$ is a protein complex formed by Apical Membrane Antigen 1 (AMA1) and Rhoptry Neck Proteins (RON) 2, 4, 5 [1]. RON2 homologs are present in various species of the Apicomplexa phylum [1-4]. In Plasmodium vivax and Plasmodium falciparum, this protein is expressed in schizonts and secreted by the rhoptries at the end of the erythrocytic cycle [3]. Although the interaction between RON2 and AMA1 is essential to the invasion process [5-7], the mechanism of interaction is not well understood. RON2 is transferred to the red blood cell (RBC) 
membrane and adopts a surface-exposed loop that binds to a hydrophobic groove in AMA1, which is secreted by micronemes at the parasite surface $[1,7,8]$. This interaction triggers the junction formation and the invasion process. In the same microenvironment of erythrocyte invasion, anti-parasite acquired immunity occurs largely through the recognition of blood stage antigens expressed by the merozoite.

Thus, merozoite proteins are important targets and promising candidates for a malaria vaccine [9-12]. However, the development of a vivax malaria vaccines is still at an initial stage, and blood stage antigens that could be novel vaccine candidates are not well known [13]. RON2 is present in various Plasmodium species and is likely exposed to the host immune system during erythrocyte invasion, making this protein a potential target for antibody-mediated protective immunity and vaccine development.

The genetic diversity of a candidate antigen becomes relevant when pursuing an efficient protective immune response. The expression of $P$. vivax proteins with a high degree of polymorphism and the corresponding strainspecific immune response represent major obstacles to vaccine development $[14,15]$. The high antigenic diversity of the parasite explains the slow development of naturally acquired immunity [16]. Thus, repeated antigen exposure over several years is necessary to generate a great repertoire of antibodies against different serotypes in an endemic area [17].

In malaria endemic regions, individuals are naturally exposed to malaria, and therefore, they produce specific immune responses against several strains. The acquired immunogenicity is generally short-lived, strain-specific and developed gradually after repeated infections [1719]. This immunity can restrain parasitaemia, protecting the individual against severe disease and decreasing the risk of mortality.

In this work, the polymorphism patterns of $P$. vivax RON2 and the naturally acquired antibody responses to this antigen were characterized genetic diversity analysis and immunogenicity to PvRON2 lays a foundation for the potential future design and development of an effective PvRON2-based malaria vaccine.

\section{Methods}

\section{Study area, population and sample collection}

To evaluate the genetic diversity of pvron 2 (sequence from nucleotide 5481-6240 from the pvron 2 gene), a total of 36 P. vivax isolates were collected from patients in Manaus, Amazonas-Brazil at Fundação de Medicina Tropical Dr. Heitor Vieira Dourado (FMT-HVD) (CAAE0044.0.114.000-11/CAAE 54234216.1.0000.0005) and in Mâncio Lima and Acrelândia, Acre State (Fig. 1) between
2011 and 2013 and in 2015 (936/CEP, 2010 and 1169/ CEPSH, 2014).

Humoral immune responses to a recombinant PvRON2 (rPvRON2) antigen in Manaus (- 03 $06^{\prime} 26^{\prime \prime}$; $\left.60^{\circ} 01^{\prime} 34^{\prime \prime} \mathrm{W}\right) \quad$ (CAAE-0044.0.114.000-11/CAAE $54234216.1 .0000 .0005)$ and in Itaituba $\left(04^{\circ} 16^{\prime} 34^{\prime \prime} \mathrm{S}\right.$; $55^{\circ} 59^{\prime} 01^{\prime \prime}(\mathrm{W})$, a gold mining area located in the most southwestern part of the State of Pará, were assessed (Fig. 1). The Annual Parasite Incidence designates Itaituba as an area with a high risk of malaria transmission (102.0 cases/1000 inhabitants per year) (CAAE001.219.346-15). In contrast, Manaus presents a low malaria transmission risk ( 5.8 cases $/ 1000$ inhabitants per year).

Samples of peripheral blood were collected from the following three groups: (1) individuals acutely infected with $P$. vivax in Itaituba $(\mathrm{n}=93)$; (2) individuals who were not infected but previously had malaria $(\mathrm{n}=97)$; and (3) acutely infected individuals from Manaus $(n=124)$. All individuals were previously diagnosed by thick blood film microscopy screens, and $P$. vivax mono infection was also determined by nested PCR, as previously described [20].

\section{Target sequence}

The PvRON2 gene has a length of $6612 \mathrm{bp}$. A synthetic gene fragment was designed based on the nucleotide sequence of the pvron 2 Sal1 strain (PlasmoDB PVX_117880). This sequence is only partially located in the PvRON2 region that binds to AMA1 during the MJ formation. Nevertheless, the selected region presented a high score in the antigenicity analysis, according to the Kolaskar and Tongaonkar method, using the program Immune Epitope database and Analyses Resource (IEDB Analysis Resource) [21].

\section{Amplification and sequencing of pvron2 from Amazonian isolates}

Genomic DNA from P. vivax isolates collected in Manaus was extracted using standard phenol-chloroform methods [22]. The DNA templates from the isolates collected in Mâncio Lima and Acrelândia were isolated from $200 \mu \mathrm{L}$ of whole blood using QIAamp DNA blood kits (Qiagen, Hilden, Germany), with a final DNA elution volume of $200 \mu \mathrm{L}$, according to the manufacturer's instructions. DNA samples were stored at $-20^{\circ} \mathrm{C}$ prior to use.

DNA samples of $P$. vivax were used as the template for the amplification of the pvron $2759 \mathrm{pb}$ sequence. Three DNA fragments were PCR-amplified to obtain the complete fragment. The oligonucleotide sequences used in this study are listed in Additional file 1. The PCR conditions used to amplify fragments one and three were as follows: 1 cycle of $5 \mathrm{~min}$ at $95^{\circ} \mathrm{C}$ followed by 35 cycles of $30 \mathrm{~s}$ at $95^{\circ} \mathrm{C}, 45 \mathrm{~s}$ at $60{ }^{\circ} \mathrm{C}, 1 \mathrm{~min}$ at $72{ }^{\circ} \mathrm{C}$ 


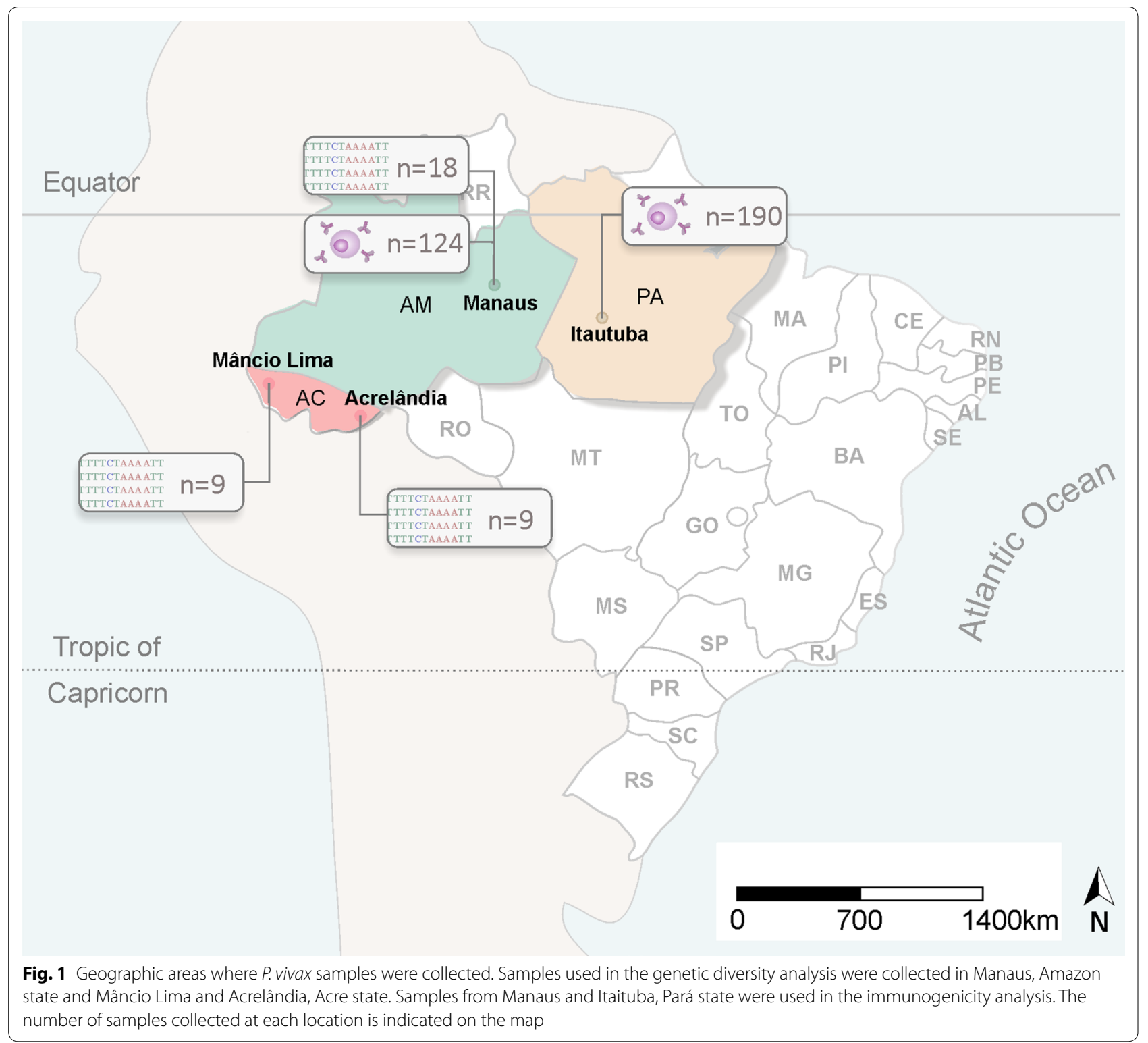

and a final cycle of $5 \mathrm{~min}$ at $72{ }^{\circ} \mathrm{C}$. To amplify fragment two, the PCR conditions were as follows: 1 cycle of $5 \mathrm{~min}$ at $94{ }^{\circ} \mathrm{C}$ followed by 35 cycles of $30 \mathrm{~s}$ at $94{ }^{\circ} \mathrm{C}$, $30 \mathrm{~s}$ at $60^{\circ} \mathrm{C}, 45 \mathrm{~s}$ at $72{ }^{\circ} \mathrm{C}$ and a final cycle of $5 \mathrm{~min}$ at $72{ }^{\circ} \mathrm{C}$. The reactions were performed using a reaction mixture containing $2.5 \mathrm{mM} \mathrm{MgCl}_{2}, 0.5 \mathrm{mM}$ each dNTP (Invitrogen), 0.5 units of Platinum Taq polymerase (Invitrogen) and $1 \mu \mathrm{M}$ of each oligonucleotide primer in a final volume of $50 \mu \mathrm{L}$. The purified PCR product was sequenced using 3730xl DNA Analyzer (Applied Biosystems).

\section{Sequence alignment and analysis}

Amplified pvron 2 sequences from 36 Brazilian Amazon isolates were analysed. The fragments were assembled using CodonCode Aligner v. 6.0.2, and the sequence data was deposited in the GenBank (accession numbers are listed in Additional file 2). Single nucleotide polymorphisms (SNPs) were identified in the alignment of 103 pvron 2 sequences from 7 other countries (Thailand, Mexico, Mauritania, China, Peru, Colombia and North Korea), which were previously deposited in the PlasmoDB [23], and GenBank [24] databases. All sequences 
were compared to the $P$. vivax reference sequence Sal-1 strain (PlasmoDB: PVX117880) using CLC Sequence Viewer 7.

\section{Expression, purification and confirmation of the PvRON2 protein in Escherichia coli}

A PvRON2 gene fragment encoding amino acid residues 1828-2080 was obtained from the genomic DNA of the $P$. vivax Sal I strain. This fragment was codon optimized for $E$. coli and subsequently cloned into the pGEX 4T-1 vector (synthetized by GenScript USA Inc. Piscataway, New Jersey). For recombinant GST-PvRON2 protein expression, the pGEX4T-1_pvron 2 plasmid was transformed into competent STAR BL21(DE3) E. coli cells by heat shock [25]. Then, the bacteria cultures were grown according to the manufacturer's instructions. Briefly, $100 \mathrm{~mL}$ of overnight culture was transferred into $3 \mathrm{~L}$ of LB containing ampicillin $(100 \mu \mathrm{g} / \mathrm{mL})$ and incubated at $37^{\circ} \mathrm{C}$ with shaking. When the culture reached an $\mathrm{OD}_{600}$ of 0.6 , protein expression was induced by the addition of $0.8 \mathrm{mM}$ IPTG for $4 \mathrm{~h}$ at $37{ }^{\circ} \mathrm{C}$. The culture was pelleted by centrifugation $(6000 \times g, 10 \mathrm{~min})$, resuspended in $25 \mathrm{~mL}$ of lysis buffer $(10 \mathrm{mM}$ Tris $\mathrm{HCl} \mathrm{pH} \mathrm{8.0,150} \mathrm{mM}$ $\mathrm{NaCl}, 1 \mathrm{mM}$ EDTA) in the presence of $1 \times$ Complete Protease Inhibitor Cocktail (Roche, Mannheim, Germany) and incubated for $1 \mathrm{~h}$ on ice. The sample was then disrupted using an M-110 L Pneumatic High Shear Fluid Processor (Microfluidcs). Next, cell fragments were pelleted by $30 \mathrm{~min}$ of centrifugation at $10,000 \times \mathrm{g}$. Finally, the supernatant was collected and analysed using SDS-PAGE and Western Blot to confirm protein expression.

\section{Measurement of antibody reactivity to rPvRON2}

Naturally, acquired IgG and IgM antibodies against rPvRON2 were measured in the plasma samples by direct enzyme-linked immunosorbent assay (ELISA). Plasma samples from infected $(n=93)$ and non-infected $(n=97)$ individuals from Itaituba, and infected individuals from Manaus $(n=124)$ were evaluated for the presence of IgG antibodies to rPvRON2. The same groups were evaluated for the presence of IgM antibodies to rPvRON2, as follows: infected individuals from Itaituba $(\mathrm{n}=56)$, noninfected individuals from Itaituba $(\mathrm{n}=97)$, and infected individuals from Manaus $(n=68)$. Samples from noninfected individuals from a non-endemic region were used as negative controls $(\mathrm{n}=21)$.

High-protein binding 96-well ELISA plates were coated with $50 \mu \mathrm{L}$ of $\mathrm{rPvRON} 2$ at $5 \mu \mathrm{g} / \mathrm{mL}$ in $0.05 \mathrm{M}$ carbonate-bicarbonate, $\mathrm{pH} 9.6$, overnight at $4{ }^{\circ} \mathrm{C}$. Then, plasma samples $(100 \mu \mathrm{L})$ diluted 1:100 were added to each well and incubated for $1 \mathrm{~h}$ at room temperature. For the detection of bound antibodies, the samples were incubated with a 1:2000 dilution of peroxidase-conjugated goat anti-human IgG or IgM (Sigma). The optical density (OD) was measured at $490 \mathrm{~nm}$ using CLARIOstar data analysis.

To avoid a bias in the results caused by the possible reactivity of the GST tag during protein expression, the excess band size was measured on the SDS PAGE gel using ImageJ. To calculate the protein surplus, plates were coated with GST. The values obtained in each sample reaction to GST were subtracted from the value obtained in the reaction against PvRON2.

All plates tested were normalized using the values of the anti-GST controls (4 well per plate). The cutoff value was calculated as the mean plus three standard deviations of the negative control. The reactivity indices (RIs) were obtained from the ratio of the absorbance values of each sample and the cutoff value. The prevalence of IgG and IgM against the rPvRON2 antigen was considered positive if the (RI) values were higher than 1.0.

The detection of IgG subclasses was performed as mentioned above, except that IgG1 (HRP), IgG2 (HRP), IgG3 (HRP) and IgG4 (HRP) specific secondary monoclonal mouse anti-human antibodies (abcam) diluted 1:2000 were used. The results were expressed as the $\mathrm{RI} \pm \mathrm{SEM}$ (standard error of the mean).

\section{Measuring cytokine levels}

The plasma levels of the cytokines IL-6, IL-10, IFN- $\gamma$ and TNF were quantified by flow cytometry using the BD IL-6, IL-10, IFN- $\gamma$, TNF Human Flex Set (BD Bioscience Pharmingen, San Diego, Ca, USA) following the instructions provided by the manufacturer. Data analyses were performed using the FACSDiva software (BD Biosciences, San Jose, CA, USA). The cytokine concentrations in each sample were determined based upon standard curves. The plasma cytokine concentrations for each sample were extrapolated from the standard curves, and the data were expressed as $\rho g / \mathrm{mL}$.

\section{Correlation coefficients and network analysis}

As the antibody levels were not normally distributed, nonparametric tests were used. Spearman's correlation was applied to assess the association between antibody levels with the following parameters: age, parasitaemia, platelets, RBC, haematocrit, haemoglobin, IL-6, IL-2, IL-10, IL-4, TNF and IFN- $\gamma$. Correlation networks were generated by the analysis of relationships among each mediator measured in the plasma samples. The systemic levels of each mediator were input in the R software (v. 3.4.3). Initially, pairwise Spearman's correlation coefficients were calculated using the $\mathrm{R}$ programming language. Along with the Spearman rank-order correlation coefficient, the $\mathrm{p}$ value to test for non-correlation was evaluated using $\mathrm{p} \leq 0.05$ as a cutoff. Moreover, 
based on the Spearman correlation coefficient, the same software was applied to identify links (edges) of interaction between the mediators (nodes). The correlation strength is represented by edge transparency and width; positive correlations are represented by red edges, and negatives correlations are represented by blue edges. Following this approach, each mediator was selected as a target, and the R software was used to perform a search within the other mediators for those that were associated with the target, in terms of correlation strength. As a result, the features related to the selected target were linked. This process was repeated for each mediator, and the result was the inferred network among the input values. To analyse the structure of the networks; the graphics of the network analysis were customized using Cytoscape software (v 3.5.1). The prefuse force-directed layout was applied, which, in the equilibrium states for the system of forces, represents the correlation strength, the edges with uniform length, and nodes that are not connected by an edge tend to be drawn further apart.

\section{Statistical analysis}

Fisher's test was applied to compare the prevalence of positive responses, and differences between medians and cytokine levels were assessed with the Mann-Whitney U-test using GraphPad Prism. A p-value of $p<0.05$ was considered significant.

\section{Results}

\section{PvRON2 is a conserved antigen}

A total of 36 pvron 2 sequences corresponding to the amino acid residues $1828-2080$, based on the $P$. vivax reference sequence Sal-1 (PlasmoDB PVX_117880), were generated from gDNA samples of $P$. vivax-infected individuals from Manaus, Mâncio Lima and Acrelândia in the Amazon region (Fig. 1). The Brazilian sequences were then compared with 103 other sequences from eight countries that were previously deposited in GenBank and PlasmoDB (Additional file 3).

Sequence analysis revealed that pvron $2_{5482-6240 \mathrm{nt}}$ is highly conserved among $P$. vivax isolates worldwide. Only two synonymous nucleotide substitutions were found among 118 sequences. One of these substitutions was observed at position $76 \mathrm{nt}$ and was detected in 47 isolates from six out of the nine countries analysed. Another substitution was found at position $228 \mathrm{nt}$ in one sequence from Peru (Additional file 3B). Non-synonymous substitutions were not detected in any of the analysed sequences, indicating that pvron 2 in a highlyconserved protein (Additional file 3A).

\section{Demographic profiles of individuals from Itaituba}

In this study, a total of 93 infected and 97 non-infected individuals from Itaituba were analysed. A higher frequency of infected individuals was observed among males (80.6\%) compared with females (19.4\%). The median age of infected individuals was 32 years old, which was significantly lower than that of uninfected individuals (43 years old). In the non-infected group, the majority of individuals reported suffering more previous episodes of malaria compared to the infected group of patients. Malaria infection significantly affected the prevalence of anaemia $(24.7 \%$ vs $9.3 \%$ for the infected and non-infected groups, respectively) and thrombocytopaenia $(79.6 \%$ vs 19.6 for the infected and non-infected groups, respectively) (Table 1).

\section{Naturally acquired antibody response towards rPvRON2}

Recombinant PvRON2 was successfully expressed in $E$. coli STAR cells as a GST fusion protein. The purity and quality of the recombinant protein were verified by SDSPAGE, which revealed a single band of approximately $56 \mathrm{kDa}$. The generated recombinant protein (rPvRON2) was recognized by the plasma from $P$. vivax-infected patients, confirming its immunoreactivity (Additional file 4).

Table 1 Epidemiological parameters of the subjects exposed to malaria in the gold mining region (Itaituba)

\begin{tabular}{llll}
\hline Characteristics & Infected (93) & Non-infected (97) & $\mathbf{p}^{*}$ \\
\hline Gender, male (\%) & 80.6 & 62.4 & 0.001 \\
Age, median years (range) & 32 & 43 & $<0.0001$ \\
Past malaria infections & & & \\
$\geq 4$ & 41.6 & 74.2 & $<0.0001$ \\
$<4$ & 58.4 & 25.8 & \\
Haemoglobin (g/dL) & 14.0 & 14.3 & 0.008 \\
Anaemia (\%) & 24.7 & 9.3 & \\
Platelets (cells/mm ${ }^{3}$ ) & 104,000 & 190,000 & $<0.0001$ \\
Thrombocytopaenic (\%) & 79.6 & 19.6 & \\
Cytokines (pg/mL) & & & 0.0002 \\
INF- $\gamma$ & 2.04 & 0 & $<0.0001$ \\
IL-6 & 24.38 & 3.02 & $<0.0001$ \\
IL-10 & 81.95 & 0.22 & 0.09 \\
IL-2 & 0 & 0 & 0.63 \\
IL-4 & 0 & 0 & 0.03 \\
TNF-a & 3.09 & 0.86 & \\
\hline
\end{tabular}

Anaemia was considered positive when haemoglobin levels were under $13 \mathrm{~g} / \mathrm{dL}$ for men or under $12 \mathrm{~g} / \mathrm{dL}$ for women

Thrombocytopaenia was defined as a platelet count of less than $150 \times 10^{3} \mathrm{per}$ $\mu \mathrm{L}$

* $p$-values were calculated from the Chi-squared test for qualitative variables or the Mann-Whitney test for non-parametric continuous variables 
To evaluate PvRON2 as a vaccine candidate, the immune response towards this antigen was assessed. A total of 190 individuals from Itaituba (93 infected and 97 non-infected) were evaluated for the presence of a naturally acquired IgG antibody response to rPvRON2, and 153 individuals (56 infected, 97 non-infected) were evaluated for an IgM response. In addition, 124 and 68 infected patients from Manaus were also analysed for IgG and IgM responses, respectively, to evaluate possible regional differences among infected patients.

IgG antibodies naturally acquired were prevalent in $19 \%(18 / 93)$ of the samples from infected individuals from Itaituba. The reactivity indices of anti-PvRON2 IgG antibodies were similar between infected patients from Itaituba and Manaus. While the individuals from Itaituba presented a prevalence of $19 \%$, individuals from Manaus showed a prevalence of 27\% (34/124) (Fig. 2a). Finally, the highest level of IgG response was found in the non-infected individuals from Itaituba (Fig. 2b), with a prevalence of $33 \%$ (32/97). The presence of naturally acquired antibodies towards rPvRON2 in plasma samples from Itaituba in individuals who had been infected with malaria in the past but exhibited no parasitaemia at the moment of blood harvest was significantly higher compared with infected individuals $(\mathrm{p}=0.047)$, suggesting a possible maintenance of the PvRON2-specific antibody response.
Some of the individuals with a positive reactivity index, both from Manaus and Itaituba $(n=59)$, were isotyped to detect IgG subclasses (IgG, IgG2, IgG3, IgG4). The results revealed that $22.03 \%$ of individuals exhibited anti-IgG1, $10.16 \%$ exhibited anti-IgG2, 13.55\% exhibited anti-IgG3 and only $1.69 \%$ exhibited anti-IgG4 antibodies (Fig. 3).

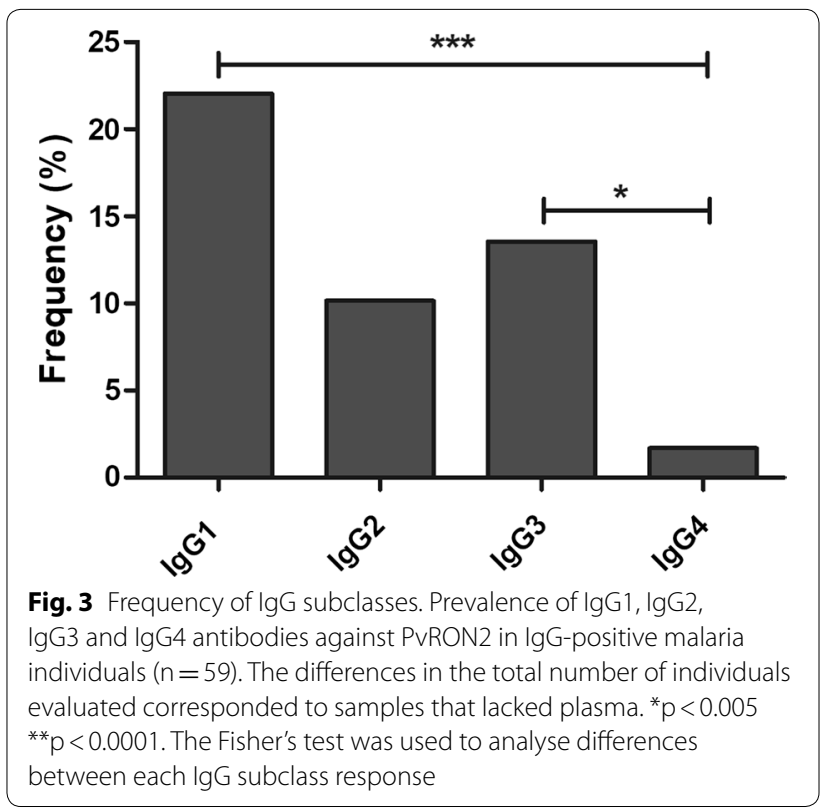

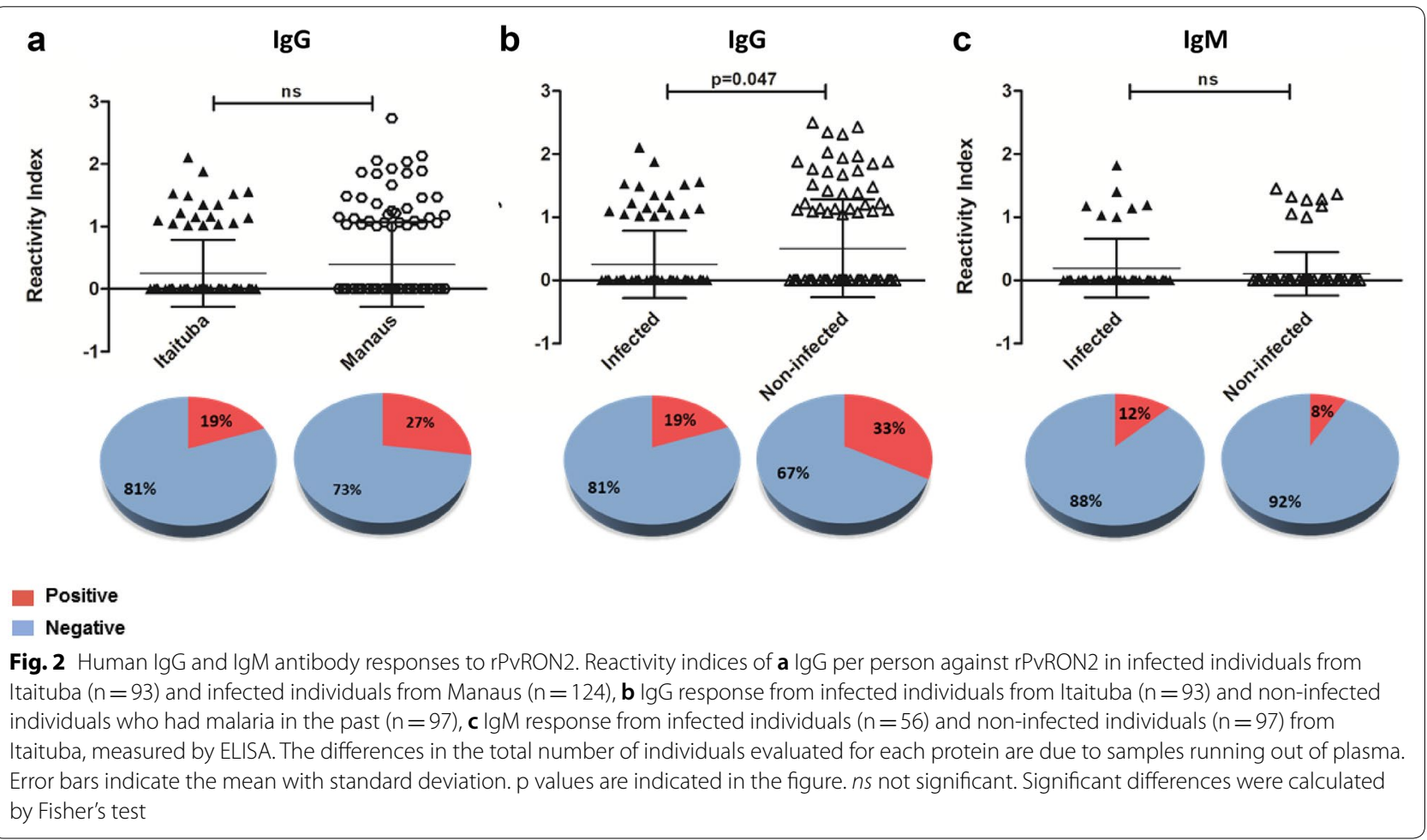


The prevalence of IgM antibodies was lower compared to IgG, as follows: $12.5 \%$ (7/56) for patients from Itaituba and $8 \%(8 / 97)$ for non-infected individuals from Itaituba (Fig. 2c). In samples from Manaus, only 4\% (3/68) of individuals were positive for an $\operatorname{IgM}$ antibody response to rPvRON2.

\section{Cytokine levels and network analysis}

To evaluate a possible correlation between the levels of cytokines and several parameters, the plasma levels of TNF, IFN- $\gamma$, IL-2, IL-4 IL- 6 and IL-10 in all individuals from Itaituba were measured (Table 1). The cytokine levels were found to be positively associated with age, parasitaemia, platelets, $\mathrm{RBC}$, haematocrit and haemoglobin; one significantly negative correlation was detected between rPvRON2 reactivity index (RI) and IL-2 $(\mathrm{p}<0.05 ; \mathrm{r}=-0.58)$ in infected individuals (Fig. 4a, b). No significant correlations were observed between the rPvRON2 reactivity index and the plasma levels of TNF, IFN- $\gamma$, IL-6, or IL-10 when analysed in both the non-infected and infected groups. In addition, a positive significant correlation between RI and age was observed in non-infected individuals (Fig. 4c, d).

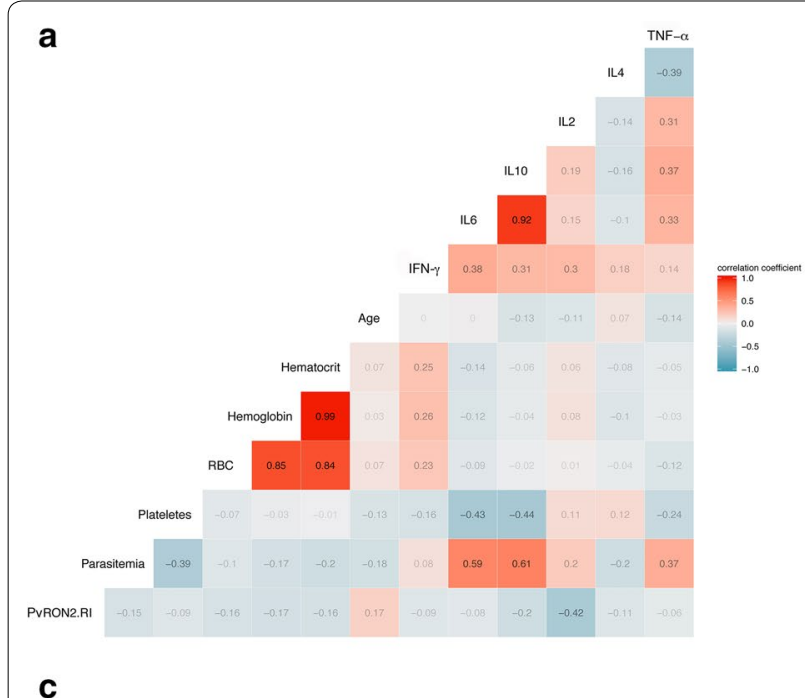

b

C

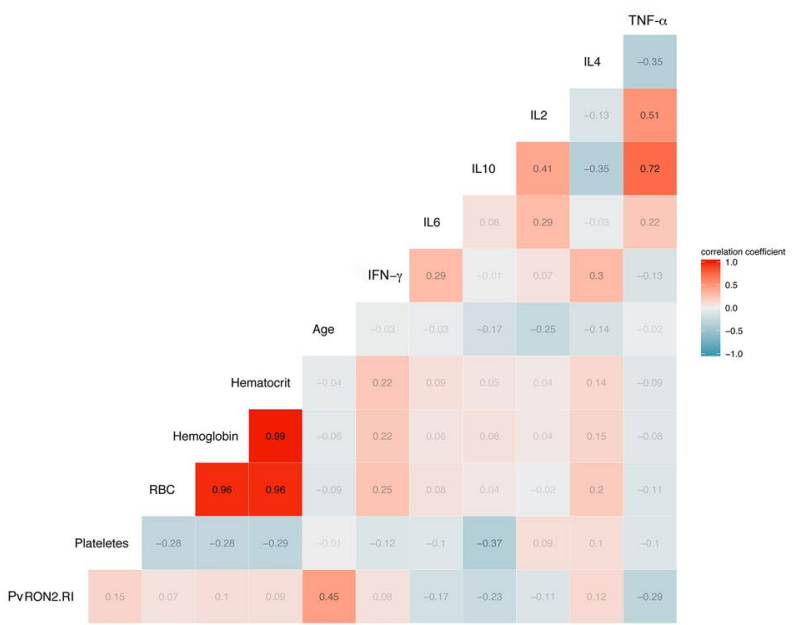

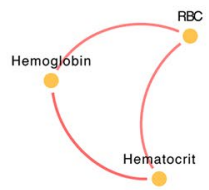

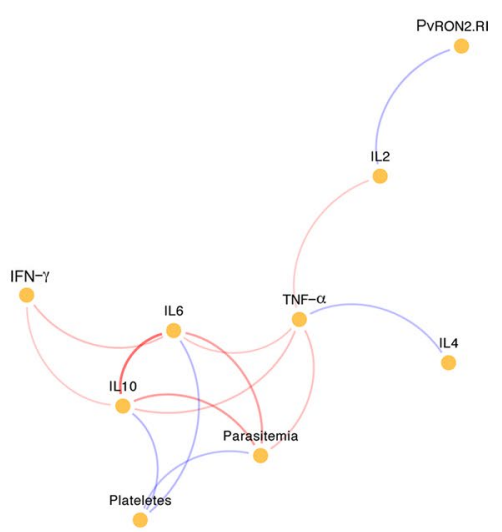

d

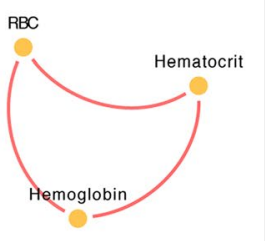

Fig. 4 Multivariate correlation coefficients and networks in infected and non-infected individuals from Itaituba. Spearman's correlation was applied to assess the association between the PVRON2 reactivity index with age, parasitaemia, platelets, RBC, hematocrit, hemoglobin, IL-6, IL-2, IL-10, $\mathrm{IL}-4, \mathrm{TNF}-\mathrm{a}$ and IFN- $\gamma$, and correlation networks were generated by the analysis of the relationship among each mediator measured in the plasma samples in $\mathbf{a}, \mathbf{b}$ the infected group and $\mathbf{c}$, $\mathbf{d}$ the non-infected group. Each connecting line (edge) represents a significant interaction $(\mathrm{p}<0.05$ ) detected by the network analysis using the R software. Correlation strength is represented by the tile or edge color transparency and width. Positive correlations are represented with red tiles/edges, and negatives correlations are represented with by blue tiles/edges 
When cytokine levels were compared between individuals with a positive RI and a negative RI, a significant difference for both IL-2 $(\mathrm{p}=0.019)$ and IL-10 $(\mathrm{p}=0.006)$ was observed; individuals with anti-PvRON2 antibodies had lower levels of IL-2 and IL-10 when compared to individuals with a negative reactivity index (Fig. 5).

\section{Discussion}

Many currently identified vaccine candidates present a high degree of polymorphism [26-28]. Selective pressure exerted by the host immune system, leads to the establishment of allele variants in the parasite population, reflected on the level of polymorphism rate [29]. One of the greatest challenges in the development of effective vaccines which are capable of generating an efficient antigen-specific immune response is the high level of polymorphic sequences. Genetic variability has been assessed in many important $P$. vivax vaccine candidates, such as CSP [30, 31], DBP [32, 33], MSP-1 [33, 34], MSP-3 alpha [35], MSP-4 [36], MSP-5 [37], MSP-7 [38], MSP-9 [39], RAP-1 [40], RAP-2 [40], Pvs48/45 [41], PvGCS1 [42], TRAP [43], and PvCelTOS [44].

Proteins involved in the MJ complex formation have also been characterized. PvAMA1 has been analysed and characterized in multiple studies on field isolates from different endemic area [29, 33, 45, 46]. PvAMA1 is a highly polymorphic antigen $[29,45,46]$. The complete AMA1 gene contains an ectodomain with three distinct subdomains (DI, DII and DIII) separated by disulfide bonds between the cysteine residues [47]. DI exhibits a higher mutation ratio and level of diversifying selection in P. falciparum [48, 49], whereas most of the polymorphic sites of AMA1 occur in domains I and II in P. vivax isolates $[46,50,51]$.

PfRON2 and PvRON2 interact with a hydrophobic groove in AMA1 $[52,53]$. It has been recently reported that PvRON2-RI (957-1288 AA) [52-54] and PvRON2RII (1850-2085 AA) [54] bind to PvAMA1 DI [52, 54] with high affinity [54], and in both $P$. falciparum and $P$. vivax species it was observed that the residue Tyr251, which was reported to be essential for RON2 binding [7, $55]$, is conserved [46, 55].

Among the RON proteins belonging to the invasion complex, PvRON4 and PvRON2 have been characterized regarding polymorphisms [56-58]. PvRON4 is conserved, with a low number of SNPs. However, there exist many haplotypes, due to the presence of tandem repeats in the $\mathrm{N}$-terminal region. However, the central and C-terminal regions are highly conserved, likely because they are under functional constraint [58].

Certain pvron 2 sequences described exhibit a high level of conservation in specific regions [56, 57]. Here, a fragment corresponding to 5482-6240 nt, which encodes
PvRON21828-2080AA was analysed. This region was chosen based on the following two characteristics: its functional importance to the MJ formation during invasion, given that this region is partially located in the RII region of PvRON2, and its antigenicity, which was predicted using the IEDB Analysis Resource.

Polymorphisms in PvRON2 ${ }_{1828-2080}$ were identified in samples collected from individuals infected with malaria from three different regions of the Brazilian Amazon, and these samples were compared with isolates from other countries. The alignment of 36 nucleotide sequences from the Brazilian isolates revealed only one SNP, and when these sequences were compared with other sequences deposited in PlasmoDB and GenBank, two SNPs were identified with no amino acid changes. The high degree of conservation of this sequence is likely due to the functional role of the interaction between AMA1 and RON2 during the MJ formation [6, 7].

In addition to evaluating the genetic variation of antigens, analysis of the natural immune response is essential for vaccine development. Given the importance of PvRON2 during the invasion process, which makes this protein a potential target of the immune system, in this study was analysed the prevalence of antibodies from infected individuals from two different endemic areas in the Brazilian Amazon region (Itaituba, Pará State and Manaus, Amazonas State).

Samples from Itaituba were collected from mining regions with a high migration rate of workers who were often previously exposed to the malaria vector. Massive human influx and deforestation have greatly impacted the ecosystem, which has promoted a greater proliferation of mosquitoes, boosting the number of malaria cases [59-61]. In contrast, in the Manaus region, malaria transmission is characterized by the migration of people from rural to urban/peri-urban areas [61]. However, when the natural acquired humoral response was evaluated, there were no significant differences in the prevalence of PvRON2 IgG antibodies between the samples from the two regions. Furthermore, the analysis of IgG subclasses in a portion of the sample group $(n=59)$, including samples from both regions, revealed a significantly higher prevalence of IgG1 and IgG3. These antibodies are predominant in naturally acquired immune responses against other Plasmodium antigens in malaria endemic regions [62-64].

Subsequently, infected patients from Itaituba were compared with a group of non-infected individuals from the same region. Interestingly, this analysis revealed that non-infected individuals had a significantly higher IgG response, indicating a longlasting immune response against the PvRON2 antigen. Because no precise information was available, it 
Bittencourt et al. Malar J

(2018) 17:401

Page 9 of 13

a

IL-2

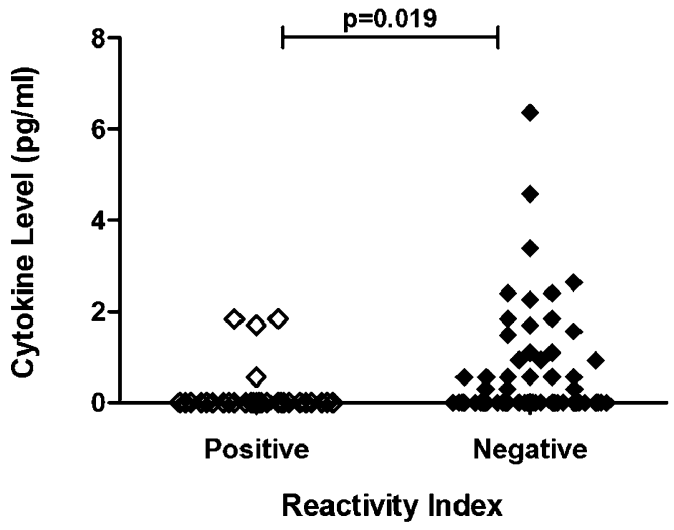

C

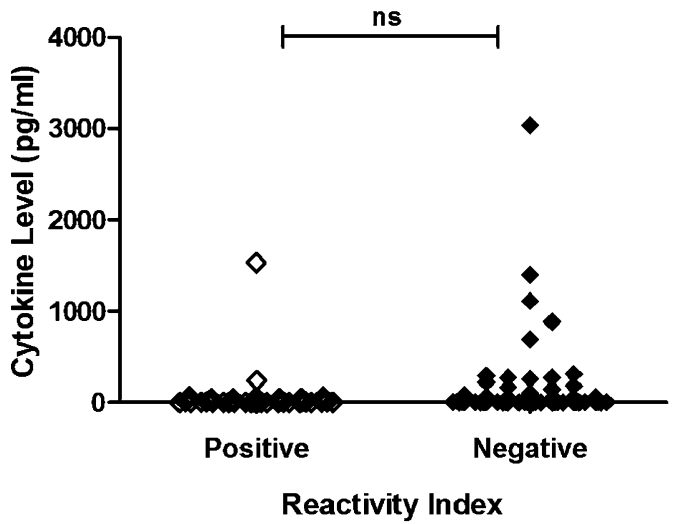

e

IF N- $\gamma$

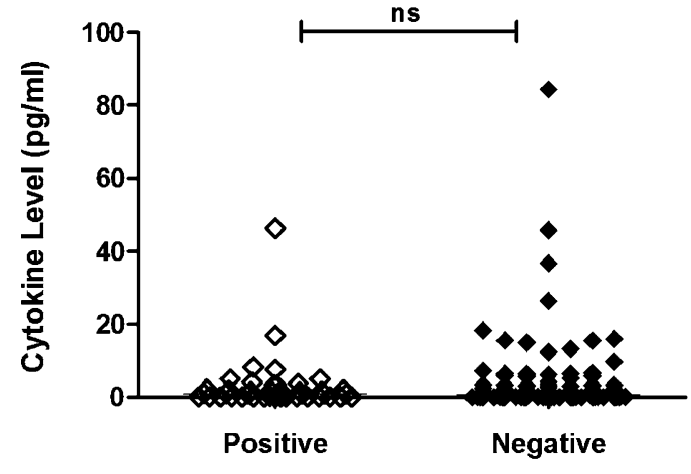

Reactivity Index b

IL-10

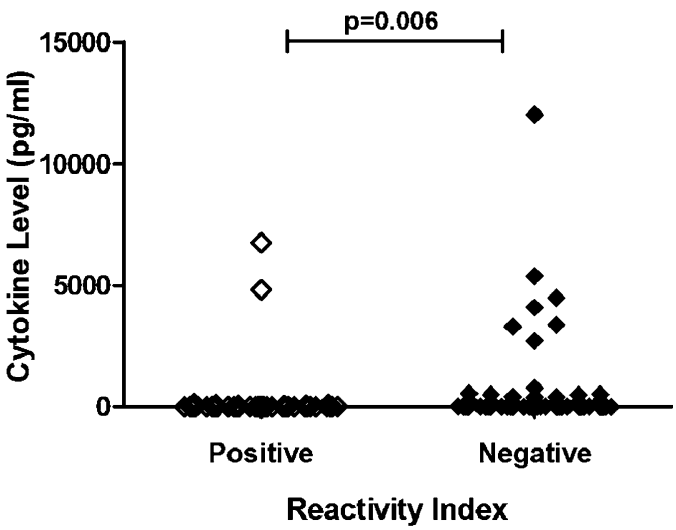

d

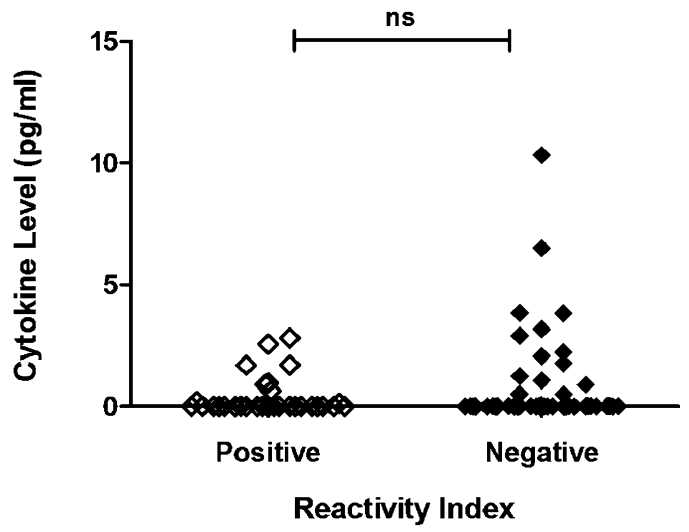

f

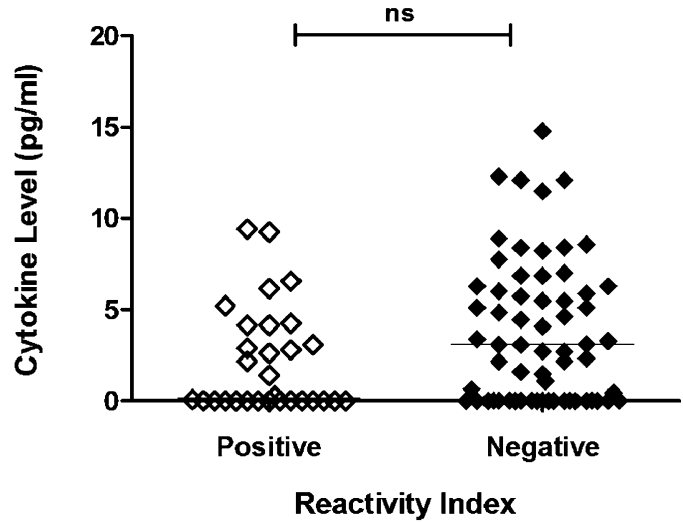

Fig. 5 Cytokine Levels and the immune response to rPvRON2. Plasma samples from Itaituba were divided in two groups, with positive (RI > 1; $n=41)$ and negative $(R L<1 ; n=103)$ reactivity indices against PvRON2. The levels of the cytokines: $\mathbf{a}\|L-2, \mathbf{b}\| L-10, \mathbf{c}\|L-6, \mathbf{d}\| L-4$, e INF- $\gamma$, f TNF-a were evaluated by flow cytometry. Bars indicate the median. $p$ values are indicated in the figure Significant differences were calculated by the MannWhitney test. ns not significant 
was not possible to correlate the reactivity index to the number of previous malaria exposures.

In non-infected individuals, a positive correlation between RI and age was observed. This type of association has been described for several Plasmodium antigens [65-68], suggesting age dependence and a possible maturation of the immune system over time. A lower prevalence of IgM compared to IgG was observed in all groups. Non-infected individuals had an even lower prevalence compared with the infected individuals from Itaituba, although this difference was not significant. Low reactivity was expected, as IgM is generally produced during the first weeks after antigen recognition and decreases over time after the first activation, whereas IgG responses can increase following subsequent exposures to the antigen, often due to the humoral immunological memory [69].

The only published work evaluating PvRON2 antigenicity analysed the natural acquired immune response against four in silico-predicted B-cell epitopes of PvRON2, none of which is located in the RI or RII functional regions. Although there was an immune response against these epitopes, the response was low [70].

Higher levels of IL-10 are positively associated with high parasitaemia $[71,72]$. In the present study, individuals who exhibited a positive reactivity index against PvRON2 exhibited lower plasma levels of IL-10. However, this group consisted primarily of noninfected individuals who were previously infected with malaria (without parasitaemia). Thus, it is possible that low levels of this cytokine are associated with the low parasitaemia in this group and not directly associated with reactivity against PvRON2.

RON2 is a potential vaccine candidate, as blocking the interaction between AMA1 and RON2 inhibits erythrocyte invasion [6, 7]. Several studies have aimed to verify the efficacy of AMA1 as a vaccine candidate [11, 73-75]. However, PfAMA1 vaccines did not provide significant protection against malaria in clinical trials [75, 76]. Meanwhile, rats immunized with a PfAMA1-RON2L complex, produced qualitatively better $P$. falciparum inhibitory antibodies upon invasion of RBCs compared with IgG elicited by the formulation containing only PfAMA1 [77]. Vaccination with this complex provided significantly higher protection in mice [77] and Aotus monkeys [78] compared with a formulation with PfAMA1 alone. In addition, a study with another fragment of the PfRON2 sequence (84aa968aa) demonstrated that IgG antibodies against this sequence are associated with clinical protection [79].

\section{Conclusions}

Taken together, the findings in this study demonstrate that PvRON2 $2_{1828-2080}$ is conserved and, moreover, there is a possible persistence of the immune response against this antigen. The data presented here suggest that PvRON2 ${ }_{1828-2080}$ may be a potential candidate to overcome the antigenic diversity limitations in vaccine design in future studies.

\section{Additional files}

Additional file 1. Primer sequences used for the amplification of pvron2 (5482-6240 nt).

Additional file 2. Country, number of sequences, accession numbers and database used in the polymorphisms analyses.

Additional file 3. Multiple sequence alignment of pvron2. Alignment of representative pvron2 sequences from Brazil and Peru, compared to the P. vivax Sal-1 strain (PlasmoDB PVX_117880). (A) Nucleotide sequences of region 5.482-6.240. (B) Amino acid sequences of region 1828-2080 aa. The alignment was made using VLC Sequence Viewer 7.

Additional file 4. Expression of recombinant PvRON2 (1828-2080 aa). Expression of rPVRON2 in bacterial expression system. (A) Expression of an $~ 56$ kDa band corresponding to PVRON2 (1828-2080 aa) and the GST tag ( 26 kDa). Lane 1: Molecular marker Spectra Multicolor High Range Protein Ladder (Thermo Scientific). Lane 2: rPvRON2. (B) Lane 1: Antibody recognition using plasma from malaria positive individual. Lane 2: Negative control.

\section{Authors' contributions}

NCB, JAL, ABIES, SCPL, JCKS, CB and TSP carried out the experimental work. JLS-F, HIN, GCC, AMRSV, MVGL, MUF and RLDM participated in the data analyses and helped to draft the manuscript. NB, LA and FTMC conceived the study design and wrote the final version of the manuscript. All authors read and approved the final manuscript.

\section{Author details}

1 Laboratory of Tropical Diseases-Prof. Dr. Luiz Jacintho da Silva, Department of Genetics, Evolution, Microbiology and Immunology, University of Campinas-UNICAMP, Campinas, SP, Brazil. ${ }^{2}$ Instituto Carlos Chagas, Fundação Oswaldo Cruz - FIOCRUZ, Curitiba, PR, Brazil. ${ }^{3}$ Laboratório de Ensaios Clínicos e Imunogenética em Malária, Instituto Evandro Chagas/SVS/MS, Ananindeua, PA, Brazil. ${ }^{4}$ Instituto Leônidas \& Maria Deane, Fundação Oswaldo Cruz FIOCRUZ, Manaus, AM, Brazil. ${ }^{5}$ Fundação de Medicina Tropical-Dr. Heitor Vieira Dourado, Manaus, AM, Brazil. ${ }^{6}$ School of Pharmaceutical Sciences, University of São Paulo, São Paulo, Brazil. ${ }^{7}$ Department of Parasitology, Institute of Biomedical Sciences, University of São Paulo-USP, São Paulo, Brazil.

\section{Acknowledgements}

Not applicable.

\section{Competing interests}

The authors declare that they have no competing interests.

\section{Availability of data and materials}

Data generated or analysed during this study are included in this published article and its Additional files or are available from the corresponding author upon request.

All Brazilian pvron2 sequences were submitted to GenBank. The accession numbers for each sequence are available in Additional file 2. 


\section{Consent for publication \\ Not applicable.}

\section{Ethics approval and consent to participate}

Samples from patients in Manaus were collected in accordance with the relevant guidelines and regulations of the ethics committee of the Fundação de Medicina Tropical - Dr. Heitor Vieira Dourado (protocols CAAE-0044.0.114.00011/CAAE 54234216.1.0000.0005) after informed consent was obtained from each subject.

Blood samples from individuals in the Pará state from Itaituba were collected under the approved protocol (CAAE-001.219.346-15) with written informed consent.

Study protocols for parasite sample collection in Acre were approved by the Institutional Review Board of the Institute of Biomedical Sciences, University of São Paulo, Brazil (936/CEP, 2010 and 1169/CEPSH, 2014). Written informed consent was obtained from all patients.

\section{Funding}

This work was supported by Fundação de Amparo à Pesquisa do Estado de São Paulo (FAPESP) Grant 2012/16525-2, and fellowships grants 2015/02808-0 (to NB), 2013/25807-4 (to JAL), 2016/12855-9 (to JLS-F), 2015/20774-6 (to GCC), 2013/20509-5 (to CB). Evandro Chagas core grant (to RLDM). MUF, MVGL and FTMC are Conselho Nacional do Desenvolvimento Científico e Tecnológico (CNPq) research fellows.

\section{Publisher's Note}

Springer Nature remains neutral with regard to jurisdictional claims in published maps and institutional affiliations.

Received: 19 March 2018 Accepted: 22 October 2018 Published online: 31 October 2018

\section{References}

1. Alexander DL, Mital J, Ward GE, Bradley P, Boothroyd JC. Identification of the moving junction complex of Toxoplasma gondii: a collaboration between distinct secretory organelles. PLoS Pathog. 2005;1:e17.

2. Cao J, Kaneko O, Thongkukiatkul A, Tachibana M, Otsuki H, Gao Q, et al. Rhoptry neck protein RON2 forms a complex with microneme protein AMA1 in Plasmodium falciparum merozoites. Parasitol Int. 2009;58:29-35.

3. Arévalo-Pinzón G, Curtidor H, Patiño LC, Patarroyo MA. PvRON2, a new Plasmodium vivax rhoptry neck antigen. Malar J. 2011;10:60.

4. Ord RL, Rodriguez M, Cursino-Santos JR, Hong H, Singh M, Gray J, et al. Identification and characterization of the rhoptry neck protein 2 in Babesia divergens and Babesia microti. Infect Immun. 2016;84:1574-84.

5. Giovannini D, Späth S, Lacroix C, Perazzi A, Bargieri D, Lagal V, et al. Independent roles of apical membrane antigen 1 and rhoptry neck proteins during host cell invasion by apicomplexa. Cell Host Microbe. 2011;10:591-602

6. Srinivasan P, Yasgar A, Luci DK, Beatty WL, Hu X, Andersen J, et al. Disrupting malaria parasite AMA1-RON2 interaction with a small molecule prevents erythrocyte invasion. Nat Commun. 2013;4:2261.

7. Srinivasan P, Beatty WL, Diouf A, Herrera R, Ambroggio X, Moch JK, et al. Binding of Plasmodium merozoite proteins RON2 and AMA1 triggers commitment to invasion. Proc Natl Acad Sci USA. 2011;108:13275-80.

8. Tonkin ML, Roques M, Lamarque MH, Pugnière M, Douguet D, Crawford J, et al. Host cell invasion by apicomplexan parasites: insights from the co-structure of AMA1 with a RON2 peptide. Science. 2011;333:463-7.

9. Richards JS, Beeson JG. The future for blood-stage vaccines against malaria. Immunol Cell Biol. 2009;87:377-90.

10. de Cassan SC, Shakri AR, Llewellyn D, Elias SC, Cho JS, Goodman AL, et al. Preclinical assessment of viral vectored and protein vaccines targeting the duffy-binding protein region II of Plasmodium vivax. Front Immunol. 2015;6:348.

11. Vicentin EC, Françoso KS, Rocha MV, lourtov D, Dos Santos FL, Kubrusly FS, et al. Invasion-inhibitory antibodies elicited by immunization with Plasmodium vivax apical membrane antigen-1 expressed in Pichia pastoris yeast. Infect Immun. 2014;82:1296-307.
12. Bargieri DY, Leite JA, Lopes SC, Sbrogio-Almeida ME, Braga CJ, Ferreira LC, et al. Immunogenic properties of a recombinant fusion protein containing the C-terminal $19 \mathrm{kDa}$ of Plasmodium falciparum merozoite surface protein-1 and the innate immunity agonist FliC flagellin of Salmonella typhimurium. Vaccine. 2010;28:2818-26.

13. Mueller I, Shakri AR, Chitnis CE. Development of vaccines for Plasmodium vivax malaria. Vaccine. 2015;33:7489-95.

14. Barry $A E$, Arnott A. Strategies for designing and monitoring malaria vaccines targeting diverse antigens. Front Immunol. 2014;5:359.

15. Takala SL, Coulibaly D, Thera MA, Batchelor AH, Cummings MP, Escalante AA, et al. Extreme polymorphism in a vaccine antigen and risk of clinical malaria: implications for vaccine development. Sci Transl Med. 2009;1:2ra5.

16. Bull PC, Lowe BS, Kortok M, Molyneux CS, Newbold Cl, Marsh K. Parasite antigens on the infected red cell surface are targets for naturally acquired immunity to malaria. Nat Med. 1998;4:358-60.

17. Marsh K, Kinyanjui S. Immune effector mechanisms in malaria. Parasite Immunol. 2006;28:51-60.

18. Mueller I, Galinski MR, Tsuboi T, Arevalo-Herrera M, Collins WE, King CL. Natural acquisition of immunity to Plasmodium vivax: epidemiological observations and potential targets. Adv Parasitol. 2013;81:77-131.

19. Marsh K, Howard RJ. Antigens induced on erythrocytes by P. falciparum: expression of diverse and conserved determinants. Science. 1986;231:150-3.

20. Snounou G, Singh B. Nested PCR analysis of Plasmodium parasites. Methods Mol Med. 2002;72:189-203.

21. Kolaskar AS, Tongaonkar PC. A semi-empirical method for prediction of antigenic determinants on protein antigens. FEBS Lett. 1990;276:172-4.

22. Moll K, Ljungström I, Perlmann H, Scherf A, Wahlgren M. Methods in malaria research. 5th ed. Manassas: MR4/ATCC; 2008.

23. Aurrecoechea C, Brestelli J, Brunk BP, Dommer J, Fischer S, Gajria B, et al. PlasmoDB: a functional genomic database for malaria parasites. Nucleic Acids Res. 2009:37(Database issue):D539-43.

24. Benson DA, Karsch-Mizrachi I, Lipman DJ, Ostell J, Wheeler DL. GenBank. Nucleic Acids Res. 2005;33(Database issue):D34-8.

25. Sambrook J, Russell DW. Preparation and transformation of competent $E$. coli using calcium chloride. CSH Protoc. 2006;2006:1.

26. Ferreira MU, Kaneko O, Kimura M, Liu Q, Kawamoto F, Tanabe K. Allelic diversity at the merozoite surface protein-1 (MSP-1) locus in natural Plasmodium falciparum populations: a brief overview. Mem Inst Oswaldo Cruz. 1998;93:631-8.

27. Cole-Tobian J, King CL. Diversity and natural selection in Plasmodium vivax Duffy binding protein gene. Mol Biochem Parasitol. 2003;127:121-32

28. Escalante AA, Grebert HM, Chaiyaroj SC, Magris M, Biswas S, Nahlen BL, et al. Polymorphism in the gene encoding the apical membrane antigen-1 (AMA-1) of Plasmodium falciparum. X. Asembo Bay Cohort Project. Mol Biochem Parasitol. 2001;113:279-87.

29. Moon SU, Na BK, Kang JM, Kim JY, Cho SH, Park YK, et al. Genetic polymorphism and effect of natural selection at domain I of apical membrane antigen-1 (AMA-1) in Plasmodium vivax isolates from Myanmar. Acta Trop. 2010;114:71-5.

30. Dias S, Wickramarachchi T, Sahabandu I, Escalante AA, Udagama PV. Population genetic structure of the Plasmodium vivax circumsporozoite protein (Pvcsp) in Sri Lanka. Gene. 2013;518:381-7.

31. Zakeri S, Abouie Mehrizi A, Djadid ND, Snounou G. Circumsporozoite protein gene diversity among temperate and tropical Plasmodium vivax isolates from Iran. Trop Med Int Health. 2006;11:729-37.

32. de Sousa TN, Carvalho LH, de Brito CFA. Worldwide genetic variability of the Duffy binding protein: insights into Plasmodium vivax vaccine development. PLOS ONE. 2011;6:e22944.

33. González-Cerón L, Cerritos R, Corzo-Mancilla J, Santillán F. Diversity and evolutionary genetics of the three major Plasmodium vivax merozoite genes participating in reticulocyte invasion in southern Mexico. Parasit Vectors. 2015;8:651.

34. Gutierrez A, Vicini J, Patarroyo ME, Murillo LA, Patarroyo MA. Plasmodium vivax: polymorphism in the merozoite surface protein 1 gene from wild Colombian isolates. Exp Parasitol. 2000;95:215-9.

35. Prajapati SK, Joshi H, Valecha N. Plasmodium vivax merozoite surface protein-3 alpha: a high-resolution marker for genetic diversity studies. J Vector Borne Dis. 2010;47:85-90. 
36. Putaporntip C, Jongwutiwes S, Ferreira MU, Kanbara H, Udomsangpetch $\mathrm{R}$, Cui L. Limited global diversity of the Plasmodium vivax merozoite surface protein 4 gene. Infect Genet Evol. 2009;9:821-6.

37. Gomez A, Suarez CF, Martinez P, Saravia C, Patarroyo MA. High polymorphism in Plasmodium vivax merozoite surface protein-5 (MSP5). Parasitology. 2006;133(Pt 6):661-72.

38. Garzón-Ospina D, López C, Forero-Rodríguez J, Patarroyo MA. Genetic diversity and selection in three Plasmodium vivax merozoite surface protein 7 (Pvmsp-7) genes in a Colombian population. PLOS ONE. 2012;7:e45962.

39. Chenet SM, Pacheco MA, Bacon DJ, Collins WE, Barnwell JW, Escalante AA. The evolution and diversity of a low complexity vaccine candidate, merozoite surface protein 9 (MSP-9), in Plasmodium vivax and closely related species. Infect Genet Evol. 2013;20:239-48.

40. Garzon-Ospina D, Romero-Murillo L, Patarroyo MA. Limited genetic polymorphism of the Plasmodium vivax low molecular weight rhoptry protein complex in the Colombian population. Infect Genet Evol. 2010;10:261-7.

41. Vallejo AF, Martinez NL, Tobon A, Alger J, Lacerda MV, Kajava AV, et al. Global genetic diversity of the Plasmodium vivax transmission-blocking vaccine candidate Pvs48/45. Malar J. 2016;15:202.

42. Mehrizi AA, Dodangeh F, Zakeri S, Djadid ND. Worldwide population genetic analysis and natural selection in the Plasmodium vivax generative cell specific 1 (PVGCS1) as a transmission-blocking vaccine candidate. Infect Genet Evol. 2016;43:50-7.

43. Putaporntip C, Jongwutiwes S, Tia T, Ferreira MU, Kanbara H, Tanabe K. Diversity in the thrombospondin-related adhesive protein gene (TRAP) of Plasmodium vivax. Gene. 2001;268:97-104.

44. Rodrigues-da-Silva RN, Soares IF, Lopez-Camacho C, da Silva JHM, Perceda-Silva DS, Têva A, et al. Cell-Traversal protein for ookinetes and sporozoites: naturally acquired humoral immune response and B-Cell epitope mapping in Brazilian amazon inhabitants. Front Immunol. 2017;8:77.

45. Arnott A, Mueller I, Ramsland PA, Siba PM, Reeder JC, Barry AE. Global population structure of the genes encoding the malaria vaccine candidate, Plasmodium vivax apical membrane antigen 1 (PVAMA1). PLoS Negl Trop Dis. 2013;7:e2506.

46. Arnott A, Wapling J, Mueller I, Ramsland PA, Siba PM, Reeder JC, et al. Distinct patterns of diversity, population structure and evolution in the AMA1 genes of sympatric Plasmodium falciparum and Plasmodium vivax populations of Papua New Guinea from an area of similarly high transmission. Malar J. 2014;13:233.

47. Pizarro JC, Vulliez-Le Normand B, Chesne-Seck ML, Collins CR, WithersMartinez $C$, Hackett F, et al. Crystal structure of the malaria vaccine candidate apical membrane antigen 1. Science. 2005;308:408-11.

48. Garg S, Alam MT, Das MK, Dev V, Kumar A, Dash AP, et al. Sequence diversity and natural selection at domain I of the apical membrane antigen 1 among Indian Plasmodium falciparum populations. Malar J. 2007;6:154.

49. Cortés A, Mellombo M, Mueller I, Benet A, Reeder JC, Anders RF. Geographical structure of diversity and differences between symptomatic and asymptomatic infections for Plasmodium falciparum vaccine candidate AMA1. Infect Immun. 2003;71:1416-26.

50. Thakur A, Alam MT, Bora H, Kaur P, Sharma YD. Plasmodium vivax: sequence polymorphism and effect of natural selection at apical membrane antigen 1 (PVAMA1) among Indian population. Gene. 2008;419:35-42

51. Gunasekera AM, Wickramarachchi T, Neafsey DE, Ganguli I, Perera L, Premaratne $\mathrm{PH}$, et al. Genetic diversity and selection at the Plasmodium vivax apical membrane antigen-1 (PvAMA-1) locus in a Sri Lankan population. Mol Biol Evol. 2007;24:939-47.

52. Vulliez-Le Normand B, Saul FA, Hoos S, Faber BW, Bentley GA. Crossreactivity between apical membrane antigen 1 and rhoptry neck protein 2 in P. vivax and P. falciparum: a structural and binding study. PLOS ONE. 2017;12:e0183198.

53. Vulliez-Le Normand B, Tonkin ML, Lamarque MH, Langer S, Hoos S, Roques $\mathrm{M}$, et al. Structural and functional insights into the malaria parasite moving junction complex. PLoS Pathog. 2012;8:e1002755.

54. Bermúdez M, Arévalo-Pinzón G, Rubio L, Chaloin O, Muller S, Curtidor $\mathrm{H}$, et al. Receptor-ligand and parasite protein-protein interactions in Plasmodium vivax: analysing rhoptry neck proteins 2 and 4. Cell Microbiol. 2018;20:e12835.
55. Collins CR, Withers-Martinez C, Hackett F, Blackman MJ. An inhibitory antibody blocks interactions between components of the malarial invasion machinery. PLoS Pathog. 2009;5:e1000273.

56. Garzón-Ospina D, Forero-Rodríguez J, Patarroyo MA. Inferring natural selection signals in Plasmodium vivax-encoded proteins having a potential role in merozoite invasion. Infect Genet Evol. 2015;33:182-8.

57. Tang J, Dai Y, Zhang H, Culleton RL, Liu Y, Zhao S, et al. Positive diversifying selection on Plasmodium vivax RON2 protein. Parasitology. 2012;139:709-15.

58. Buitrago SP, Garzón-Ospina D, Patarroyo MA. Size polymorphism and low sequence diversity in the locus encoding the Plasmodium vivax rhoptry neck protein 4 (PvRON4) in Colombian isolates. Malar J. 2016;15:501.

59. Marques AC. Human migration and the spread of malaria in Brazil. Parasitol Today. 1987;3:166-70.

60. Ferreira MU, Castro MC. Challenges for malaria elimination in Brazil. Malar J. 2016:15:284.

61. Recht J, Siqueira AM, Monteiro WM, Herrera SM, Herrera S, Lacerda MVG. Malaria in Brazil, Colombia, Peru and Venezuela: current challenges in malaria control and elimination. Malar J. 2017;16:273.

62. Rouhani M, Zakeri S, Mehrizi AA, Djadid ND. Comparative analysis of the profiles of IgG subclass-specific responses to Plasmodium falciparum apical membrane antigen-1 and merozoite surface protein-1 in naturally exposed individuals living in malaria hypoendemic settings, Iran. Malar J. 2015;14:58.

63. Weaver R, Reiling L, Feng G, Drew DR, Mueller I, Siba PM, et al. The association between naturally acquired IgG subclass specific antibodies to the PfRH5 invasion complex and protection from Plasmodium falciparum malaria. Sci Rep. 2016;6:33094.

64. Taylor RR, Allen SJ, Greenwood BM, Riley EM. IgG3 antibodies to Plasmodium falciparum merozoite surface protein 2 (MSP2): increasing prevalence with age and association with clinical immunity to malaria. Am J Trop Med Hyg. 1998;58:406-13.

65. Baird JK. Age-dependent characteristics of protection v susceptibility to Plasmodium falciparum. Ann Trop Med Parasitol. 1998;92:367-90.

66. Amoah LE, Nuvor SV, Obboh EK, Acquah FK, Asare K, Singh SK, et al. Natural antibody responses to Plasmodium falciparum MSP3 and GLURP(RO) antigens are associated with low parasite densities in malaria patients living in the Central Region of Ghana. Parasit Vectors. 2017;10:395.

67. Patel P, Bharti PK, Bansal D, Raman RK, Mohapatra PK, Sehgal R, et al. Genetic diversity and antibody responses against Plasmodium falciparum vaccine candidate genes from Chhattisgarh, Central India: implication for vaccine development. PLoS ONE. 2017;12:e0182674.

68. Khosravi A, Hommel M, Sayemiri K. Age-dependent antibody response to Plasmodium falciparum merozoite surface protein 2 (MSP-2). Parasite Immunol. 2011;33:145-57.

69. Yeom JS, Kim ES, Lim KJ, Oh JH, Sohn MJ, Yoo SB, et al. Naturally acquired IgM antibody response to the $\mathrm{C}$-terminal region of the merozoite surface protein 1 of Plasmodium vivax in Korea: use for serodiagnosis of vivax malaria. J Parasitol. 2008:94:1410-4.

70. López C, Yepes-Pérez Y, Díaz-Arévalo D, Patarroyo ME, Patarroyo MA. The in vitro antigenicity of Plasmodium vivax Rhoptry Neck Protein 2 (PvRON2) B- and T-epitopes selected by HLA-DRB1 binding profile. Front Cell Infect Microbiol. 2018;8:156.

71. Zeyrek FY, Kurcer MA, Zeyrek D, Simsek Z. Parasite density and serum cytokine levels in Plasmodium vivax malaria in Turkey. Parasite Immunol. 2006;28:201-7

72. Gonçalves RM, Scopel KK, Bastos MS, Ferreira MU. Cytokine balance in human malaria: does Plasmodium vivax elicit more inflammatory responses than Plasmodium falciparum? PLOS ONE. 2012;7:e44394.

73. Gentil F, Bargieri DY, Leite JA, Francoso KS, Patricio MB, Espindola NM, et al. A recombinant vaccine based on domain II of Plasmodium vivax Apical Membrane Antigen 1 induces high antibody titres in mice. Vaccine. 2010;28:6183-90

74. Stowers AW, Kennedy MC, Keegan BP, Saul A, Long CA, Miller LH. VaCcination of monkeys with recombinant Plasmodium falciparum apical membrane antigen 1 confers protection against blood-stage malaria. Infect Immun. 2002;70:6961-7.

75. Spring MD, Cummings JF, Ockenhouse CF, Dutta S, Reidler R, Angov E, et al. Phase $1 / 2$ a study of the malaria vaccine candidate apical membrane antigen-1 (AMA-1) administered in adjuvant system AS01B or AS02A. PLOS ONE. 2009:4:e5254. 
76. Thera MA, Doumbo OK, Coulibaly D, Laurens MB, Ouattara A, Kone AK et al. A field trial to assess a blood-stage malaria vaccine. N Engl J Med. 2011;365:1004-13.

77. Srinivasan P, Ekanem E, Diouf A, Tonkin ML, Miura K, Boulanger MJ, et al. Immunization with a functional protein complex required for erythrocyte invasion protects against lethal malaria. Proc Natl Acad Sci USA. 2014;111:10311-6.
78. Srinivasan P, Baldeviano GC, Miura K, Diouf A, Ventocilla JA, Leiva KP, et al. A malaria vaccine protects Aotus monkeys against virulent Plasmodium falciparum infection. NPJ Vaccines. 2017;2:e14.

79. Richards JS, Arumugam TU, Reiling L, Healer J, Hodder AN, Fowkes FJ, et al. Identification and prioritization of merozoite antigens as targets of protective human immunity to Plasmodium falciparum malaria for vaccine and biomarker development. J Immunol. 2013;191:795-809.
Ready to submit your research? Choose BMC and benefit from:

- fast, convenient online submission

- thorough peer review by experienced researchers in your field

- rapid publication on acceptance

- support for research data, including large and complex data types

- gold Open Access which fosters wider collaboration and increased citations

- maximum visibility for your research: over 100M website views per year

At BMC, research is always in progress.

Learn more biomedcentral.com/submissions 